Journal of Applied Mathematics and Stochastic Analysis, 16:2 (2003), 97-119.

Printed in the USA (c) 2003 by North Atlantic Science Publishing Company

\title{
FRACTIONAL DIFFERENTIAL EQUATIONS DRIVEN BY LÉVY NOISE
}

\author{
V.V. ANH and R. MCVINISH ${ }^{1}$ \\ Centre in Statistical Science and Industrial Mathematics \\ Queensland University of Technology \\ GPO Box 2434, Brisbane QLD 4001, Australia \\ E-mail:v.anh@qut.edu.au,r.mcvinish@qut.edu.au
}

(Received May 2001; Revised January 2003)

\begin{abstract}
This paper considers a general class of fractional differential equations driven by Lévy noise. The singularity spectrum for these equations is obtained. This result allows to determine the conditions under which the solution is a semimartingale. The prediction formula and a numerical scheme for approximating the sample paths of these equations are given. Almost sure, uniform convergence of the scheme and some numerical results are also provided.

Key words: Stochastic Fractional Differential Equation, Anomalous Diffusion, Multifractality, Long-Range Dependence, Semimartingale Representation.
\end{abstract}

AMS (MOS) subject classification: 60G10, 60M20

\section{Introduction}

Many macroeconomic and financial time series or their transforms apparently display the characteristics of anomalous diffusion, namely long-range dependence (LRD) and heavy-tailed marginal distributions (see, for instance, Baillie [5], Ding and Granger [17], Granger and Ding [24], Comte and Renault [15, 16], Eberlein and Keller [20], Bibby and Sørensen [10], Barndorff-Nielsen [7, 6], Barndorff-Nielsen and Shephard [8, 9], Eberlain and Raible [21], Rydberg [39]).

Barndorff-Nielsen [7, 6] used discrete or continuous-type superposition of OrnsteinUhlenbeck processes with Lévy motion input to obtain a class of random processes with LRD and infinitely divisible marginal distributions, while Igloi and Terdik [25] and Oppenheim and Viano [36] obtained long memory by aggregating continuous-time short-memory Gaussian processes with random coefficients.

In a continuous-time framework, it is known that LRD can be obtained by replacing ordinary differential operators by fractional differential operators in differential equations driven by white noise (see, for example, Gay and Heyde [23], Inoue [26], Viano et al. [42], Chambers [14], Anh et al. [1], Anh and Leonenko [3]). Following this approach, Anh et al. [2] introduced a class of general fractional differential equations

${ }^{1}$ Partially supported by the Australian Research Council grant A10024117 
(FDE) driven by Lévy noise, whose solutions are obtained as convolutions of the Green functions of the corresponding deterministic FDEs with Lévy noise or stochastic path integrals with respect to Lévy processes. The main advantage of this approach is that LRD can be effected via the Green function of the fractional operator involved, while the noise term can be used to represent the effects of non-Gaussianity or multifractality. Anh et al. [2] obtained explicit results on the Green functions, correlation functions, spectra and higher-order spectra of particular forms of these FDEs. In particular, they demonstrated that these equations can be used to model the stochastic volatility of log price processes and macroeconomic processes with long memory.

In this paper, we continue this line of investigation. Some elements of the above class of FDEs, as presented in Anh et al. [2], will be recalled in the next section. Their multifractality, which is described by the corresponding singularity spectrum, is obtained in Section 3. This result provides not only some sample path properties of the FDEs but also an important device to determine the conditions under which the solution is a semimartingale. This semimartingale representation is derived in Section 4. Section 5 gives a closed-form prediction formula, while Section 6 develops a numerical scheme for approximating the sample paths of these equations. Almost sure, uniform convergence of the scheme and some numerical results will also be given.

\section{Fractional Differential Equations Driven by Lévy Noise}

Let us first recall the definitions of fractional derivative and fractional integral (see Samko et al. [40], Djrbashian [18], Podlubny [37] among others). Assuming reasonable behaviour for $f(t)$, its Riemann-Liouville fractional derivative is defined as

$$
\mathcal{D}^{\alpha} f(t)=\frac{1}{\Gamma(n-\alpha)} \frac{d^{n}}{d t^{n}} \int_{0}^{t}(t-\tau)^{n-\alpha-1} f(\tau) d \tau,
$$

$\alpha \in[n-1, n), n=1,2, \ldots$, and its Riemann-Liouville fractional integral is defined as

$$
\mathcal{I}^{\alpha} f(t)=\frac{1}{\Gamma(\alpha)} \int_{0}^{t}(t-\tau)^{\alpha-1} f(\tau) d \tau, \alpha>0
$$

In this paper, we consider fractional differential equations of the general form

$$
\begin{gathered}
\left(A_{n} \mathcal{D}^{\beta_{n}}+\ldots+A_{1} \mathcal{D}^{\beta_{1}}+A_{0} \mathcal{D}^{\beta_{0}}\right) X(t)=\dot{L}(t), \\
\beta_{n}>\beta_{1}>\ldots>\beta_{1}>\beta_{0}, n \geq 1,
\end{gathered}
$$

where $\dot{L}$ is Lévy noise. Note that Lévy noise $\dot{L}$ has the following properties:

(1) it is infinitely divisible;

(2) its probability distribution is translation invariant and

(3) $\dot{L}(t)$ and $\dot{L}(s)$ are independent if $t \neq s$ (see Mueller [33], for example).

As defined in Podlubny [37], p.150, the function $G(t-\tau)$ satisfying the following conditions 
a) $\left(A_{n} \mathcal{D}^{\beta_{n}}+\ldots+A_{1} \mathcal{D}^{\beta_{1}}+A_{0} \mathcal{D}^{\beta_{0}}\right) G(t-\tau)=0$ for every $\tau \in(0, t)$;

b) $\lim _{\tau \rightarrow t-0} \mathcal{D}^{\beta_{k}-1} G(t-\tau)=\delta_{k, n}, k=0,1, \ldots, n, \delta_{k, n}$ being the Kronecker delta;

c) $\lim _{\tau, t \rightarrow 0+, \tau<t} \mathcal{D}^{\beta_{k}} G(t-\tau)=0, k=0,1, \ldots, n-1$

is called the Green function of Eq. (2.3). Its solution in terms of the Green function can be written as

$$
X(t)=\int_{0}^{t} G(t-s) d L(s)
$$

where formally $L(t)=\int_{0}^{t} \dot{L}(s) d s$, and the stochastic integral is known to exist (Anh et al. [2]). Alternatively, the integral (2.5) may be interpreted pathwise. Let $v_{p}(f)$ be the $p$-variation of $f$; then

$$
\begin{aligned}
\|f\|_{(p)} & =v_{p}(f)^{1 / p} \\
\|f\|_{[p]} & =\|f\|_{(p)}+\sup |f| .
\end{aligned}
$$

If $G$ is of bounded $q$-variation and $L$ is of bounded $p$-variation with $q^{-1}+p^{-1}>1$ then the integral may be interpreted

- in the Riemann-Stieltjes sense whenever $G$ and paths of $L$ have no discontinuities at the same points;

- in the Moore-Pollard-Stieltjes sense whenever $G$ and paths of $L$ have no one-sided discontinuities at the same points;

- always in the sense defined by Young [43] (see Dudley and Norvaiša [19] for details).

When the integral exists, the Love-Young inequality (Theorem 2.1 of Dudley and Norvaiša $[19])$

$$
\left|\int_{0}^{t} G(t-s) d L(s)\right| \leq C_{p, q}\|G\|_{[q]}\|L\|_{(p)}
$$

holds, where $C_{p, q}=\zeta\left(p^{-1}+q^{-1}\right), \zeta(s)=\sum_{n=1}^{\infty} n^{-s}$.

We should note that the Green function $G(t), t \geq 0$, is usually defined by its Laplace transform

$$
g(p)=\int_{0}^{\infty} e^{-p t} G(t) d t, \operatorname{Re}(p)>0 .
$$

Consider the fractional integral equation

$$
X(t)+\frac{A_{n-1}}{A_{n}} \mathcal{I}^{\beta_{n}-\beta_{n-1}} X(t)+\ldots+\frac{A_{0}}{A_{n}} \mathcal{I}^{\beta_{n}-\beta_{0}} X(t)=\frac{1}{A_{n}} \mathcal{I}^{\beta_{n}-1} L(t) .
$$

with $\beta_{n} \geq 1$. The existence and uniqueness of the solution to (2.8) in the space of regulated functions can be established. In fact, let $\mathcal{W}_{p}$ be the space of functions with bounded $p$-variation; then the metric space $\left(\mathcal{W}_{p},\|.\|_{[p]}\right)$ is a Banach space (see Theorem 4.2 of Dudley and Norvaiša [19]). Noting that (2.8) is a linear integral equation with a weakly singular Volterra kernel, Theorem 10.13 of Kress [30] can be applied to get the existence and uniqueness of a solution in $\left(\mathcal{W}_{p},\|\cdot\|_{[p]}\right)$ for $\beta_{n} \geq 1$. When $L(t)$ 
is Lévy motion, it has finite $p$-variation for any $p>2$ since Lévy motion is a semimartingale. For certain Lévy motions, Bretagnolle [11] characterised the property of finite $p$-variation, $p \geq 1$, in terms of the finiteness of the integral

$$
\int_{\mathbb{R}} \min \left(1,|x|^{p}\right) \nu(d x),
$$

where $\nu(d x)$ is the Lévy measure. Using Lemma 1 of the following section, it is seen that $\mathcal{I}^{\beta} L(t)$ has bounded $p$-variation if $L(t)$ has bounded $p$-variation and $\beta \geq 0$.

We now use the Laplace transform method to obtain an explicit representation of the solution to (2.8). Let $L_{T}(t)=L(t \wedge T)$. The Laplace transform of $L_{T}$ is well defined and will be denoted by $\widetilde{L}_{T}(p)$. As $L_{T}(t)$ is bounded for all $t \in[0, \infty), X(t)$ is also bounded on this interval and hence its Laplace transform $\widetilde{X}(p)$ is well defined. Applying the Laplace transform to (2.8) we have

$$
\begin{gathered}
\widetilde{X}(p)\left(1+\frac{A_{n-1}}{A_{n}} p^{\beta_{n-1}-\beta_{n}}+\ldots+\frac{A_{0}}{A_{n}} p^{\beta_{0}-\beta_{n}}\right)=\frac{1}{A_{n}} p^{1-\beta_{n}} \widetilde{L}_{T}(p), \\
\widetilde{X}(p)=p g(p) \widetilde{L}_{T}(p) .
\end{gathered}
$$

Inverting the Laplace transform gives

$$
X(t)=G(0) L(t)+\int_{0}^{t} G^{\prime}(t-s) L_{T}(s) d s .
$$

The integration by parts formula (see Dudley and Norvaiša [19], p.67) shows the equivalence of (2.5) and (2.12) for $t<T$. We will use the representation (2.8) to study the path properties of the solution of fractional differential equations when $\beta_{n} \geq 1$. For $\beta_{n}<1$ we will interpret the solution to $(2.3)$ as $X(t)=\mathcal{D}^{1-\beta_{n}} Y(t)$, where $Y(t)$ solves

$$
A_{n} \mathcal{D}^{1} Y(t)+\ldots+A_{1} \mathcal{D}^{\beta_{1}+1-\beta_{n}} Y(t)+A_{0} \mathcal{D}^{\beta_{0}+1-\beta_{n}} Y(t)=\mathcal{D}^{1} L(t) .
$$

We next recall some basic definitions on the local scaling properties of the paths of a process $X(t)$ on some interval $[0, T]$; for futher details see, for example, Jaffard [27] or Riedi [38] . A typical feature of a fractal process $X(t)$ is that it has a non-integer degree of differentiability, characterised by its local Hölder exponent $h(t)$ defined by

$$
h(t):=\sup _{l}\left\{l:\left|X\left(t^{\prime}\right)-P_{t}\left(t^{\prime}\right)\right|<C\left|t^{\prime}-t\right|^{l}\right\}
$$

for $t^{\prime}$ sufficiently close to $t$ and $P_{t}($.$) being the Taylor polynomial of X$ at $t$. The sets

$$
E_{h}:=\{t: h(t)=h\},
$$

which form a decomposition of the support of $X$ according to its singularity exponents, can be highly interwoven and dense on $[0, T]$. The singularity spectrum of $X$ is then defined as $d(h)=\operatorname{dim}\left(E_{h}\right)$ where $\operatorname{dim}$ is the Hausdorff dimension. A process $X$ is said to be multifractal if the support of its singularity spectrum has a non-empty interior. A classical example of a multifractal process is the multiplicative cascade on the interval $[0,1]$ (Mandelbrot [32]). However, such multiplicative cascades are not suitable for the stochastic models considered here as they do not possess stationary increments and are 
only defined on some finite interval. An example of a stochastic process with stationary increments and defined on $[0, \infty)$ which is also a multifractal is Lévy motion. Jaffard [28] showed that all Lévy motions are multifractal with the exception of Brownian motion, compound Poisson processes, deterministic motion and their convolutions. The singularity spectrum of a Lévy motion without Brownian component was shown to be

$$
d(h)=\left\{\begin{array}{cc}
\gamma h, \quad h \in[0,1 / \gamma] \\
-\infty, \quad \text { elsewhere }
\end{array}\right.
$$

where $\gamma$ is given by

$$
\gamma=\inf \left\{\eta: \int_{|x|<1}|x|^{\eta} \nu(d x)\right\}
$$

and is called the upper index of the Lévy measure.

\section{Multifractality of Fractional Differential Equations}

We first give an extension of the mapping property of fractional integrals on the space $H^{\lambda}([a, b])$ of Hölder continuous functions which states that a function $\phi \in H^{\lambda}([a, b])$ is mapped into the space $H^{\lambda+\alpha}([a, b])$ by the fractional integral of order $\alpha$ (see Samko et al. [40], Theorem 3.1).

Lemma 1 Let $X(t)$ be a process with bounded sample paths. Denote the local Hölder exponents of $X(t)$ by $h_{X}(t)$. Define $Y(t)$ by

$$
Y(t)=\int_{0}^{t}(t-s)^{\alpha-1} X(s) d s
$$

with $\alpha \in(0,1)$. Then the following inequality holds:

$$
h_{Y}(t) \geq \min \left(\alpha+h_{X}(t), 1+\alpha\right) .
$$

Proof For some $t^{\prime}>t$ (the case of $t^{\prime}<t$ can be dealt with in a similar fashion),

$$
Y\left(t^{\prime}\right)-Y(t)=\int_{0}^{t^{\prime}}\left(t^{\prime}-s\right)^{\alpha-1} X(s) d s-\int_{0}^{t}(t-s)^{\alpha-1} X(s) d s .
$$

Adding and subtracting the terms $\int_{0}^{t^{\prime}}\left(t^{\prime}-s\right)^{\alpha-1} X(t) d s$ and $\int_{0}^{t}(t-s)^{\alpha-1} X(t) d s$ gives

$$
Y\left(t^{\prime}\right)-Y(t)=J_{1}+J_{2}+J_{3}
$$

where

$$
\begin{aligned}
J_{1} & =\int_{t}^{t^{\prime}}\left(t^{\prime}-s\right)^{\alpha-1}[X(s)-X(t)] d s, \\
J_{2} & =\int_{0}^{t}\left[\left(t^{\prime}-s\right)^{\alpha-1}-(t-s)^{\alpha-1}\right][X(s)-X(t)] d s, \\
J_{3} & =\alpha^{-1} X(t)\left[\left(t^{\prime}\right)^{\alpha}-t^{\alpha}\right] .
\end{aligned}
$$


We first show that $J_{3}$ is a local polynomial for $t>0$. Now,

$$
\begin{aligned}
J_{3} & =\alpha^{-1} X(t)\left[\left(t^{\prime}\right)^{\alpha}-t^{\alpha}\right] \\
& =\alpha^{-1} X(t) t^{\alpha}\left[\left(1+\frac{t^{\prime}-t}{t}\right)^{\alpha}-1\right] \\
& =\alpha^{-1} X(t) t^{\alpha} \sum_{k=1}^{\infty}\left(\begin{array}{c}
\alpha \\
k
\end{array}\right)\left(\frac{t^{\prime}-t}{t}\right)^{k} .
\end{aligned}
$$

For $\left|t^{\prime}-t\right|$ sufficiently small and $t>0, J_{3}$ is a local polynomial and so will not affect the local Hölder exponents. From the definition of local Hölder exponents for $s$ in a neighbourhood of $t$, say $[t-\delta, t+\delta]$,

$$
X(s)-X(t)=\sum_{k=1}^{N} c_{k}(s-t)^{k}+O\left(|s-t|^{h_{X}(t)-\varepsilon}\right)
$$

for any $\varepsilon>0$. Applying this to $J_{1}$, we get

$$
\begin{aligned}
J_{1}= & \sum_{k=1}^{N} c_{k} \int_{t}^{t^{\prime}}\left(t^{\prime}-s\right)^{\alpha-1}(s-t)^{k} d s+O \\
& \times\left(\int_{t}^{t^{\prime}}|s-t|^{h_{X}(t)-\varepsilon}(t-s)^{\alpha-1} d s\right) \\
= & O\left(\left|t^{\prime}-t\right|^{\alpha+1}\right)+O\left(\left|t^{\prime}-t\right|^{h_{X}(t)+\alpha-\varepsilon}\right) .
\end{aligned}
$$

For $J_{2}$, the interval of integration is divided into $\left[2 t-t^{\prime}, t\right]$ and $\left[0,2 t-t^{\prime}\right]$. Applying (3.9) to $J_{2}$ on the first interval of integration yields

$$
\begin{aligned}
J_{2}^{(1)}= & \int_{2 t-t^{\prime}}^{t}\left[\left(t^{\prime}-s\right)^{\alpha-1}-(t-s)^{\alpha-1}\right][X(s)-X(t)] d s \\
= & \sum_{k=1}^{N} c_{k} \int_{2 t-t^{\prime}}^{t}\left[\left(t^{\prime}-s\right)^{\alpha-1}-(t-s)^{\alpha-1}\right](s-t)^{k} d s \\
& +O\left(\int_{2 t-t^{\prime}}^{t}\left[\left(t^{\prime}-s\right)^{\alpha-1}-(t-s)^{\alpha-1}\right](t-s)^{h_{X}(t)-\varepsilon} d s\right) .
\end{aligned}
$$

Noting that

$$
\left|\int_{2 t-t^{\prime}}^{t}\left[\left(t^{\prime}-s\right)^{\alpha-1}-(t-s)^{\alpha-1}\right] d s\right|=\alpha^{-1}\left(2-2^{\alpha}\right)\left(t^{\prime}-t\right)^{\alpha},
$$

we get

$$
J_{2}^{(1)}=O\left(\left|t^{\prime}-t\right|^{\alpha+1}\right)+O\left(\left|t^{\prime}-t\right|^{h_{X}(t)+\alpha-\varepsilon}\right) .
$$

For the second inteval of integration,

$$
\begin{aligned}
J_{2}^{(2)} & =\int_{0}^{2 t-t^{\prime}}\left[\left(t^{\prime}-s\right)^{\alpha-1}-(t-s)^{\alpha-1}\right][X(s)-X(t)] d s \\
& =\int_{0}^{2 t-t^{\prime}}(t-s)^{\alpha-1}\left[\left(\frac{t^{\prime}-t}{t-s}+1\right)^{\alpha-1}-1\right][X(s)-X(t)] d s .
\end{aligned}
$$


Noting that for $t \geq 0,\left|(1+t)^{\alpha-1}-1\right| \leq(1-\alpha) t$ and assuming that $\alpha+h_{X}(t)-\varepsilon \leq 1$, we obtain

$$
\begin{aligned}
J_{2}^{(2)} & =O\left(\left(t^{\prime}-t\right) \int_{0}^{2 t-t^{\prime}}(t-s)^{\alpha-2}|X(s)-X(t)| d s\right) \\
& =O\left(\left(t^{\prime}-t\right) \int_{t-\delta}^{2 t-t^{\prime}}(t-s)^{\alpha-2}|s-t|^{h_{X}(t)-\varepsilon} d s+D\left(t^{\prime}-t\right)\right),
\end{aligned}
$$

where $D=\int_{0}^{t-\delta}(t-s)^{\alpha-2}|X(s)-X(t)| d s$. Therefore, if $\alpha+h_{X}(t)-\varepsilon \leq 1$,

$$
J_{2}^{(2)}=O\left(\left|t^{\prime}-t\right|^{\alpha+h_{X}(t)-\varepsilon}\right) .
$$

In view of the equations (3.8), (3.11), (3.14) and (3.19) and letting $\varepsilon \rightarrow 0$, we get $h_{Y}(t) \geq \alpha+h_{X}(t)$ if $\alpha+h_{X}(t) \leq 1$.Now, assume that $\alpha+h_{X}(t)>1$; then from (3.16),

$$
J_{2}^{(2)}=\int_{0}^{2 t-t^{\prime}} \sum_{k=1}^{\infty}\left(\begin{array}{l}
\alpha-1 \\
k
\end{array}\right)\left(t^{\prime}-t\right)^{k}(t-s)^{\alpha-1-k}[X(s)-X(t)] d s .
$$

Applying Fubini's theorem, the order of integration and summation can be changed to yield

$$
J_{2}^{(2)}=\sum_{k=1}^{\infty}\left(\begin{array}{l}
\alpha-1 \\
k
\end{array}\right)\left(t^{\prime}-t\right)^{k} \int_{0}^{2 t-t^{\prime}}(t-s)^{\alpha-1-k}[X(s)-X(t)] d s .
$$

For $k=1$,

$$
\begin{aligned}
\int_{0}^{2 t-t^{\prime}}(t-s)^{\alpha-2}[X(s)-X(t)] d s \\
\quad=\int_{0}^{t}(t-s)^{\alpha-2}[X(s)-X(t)] d s-\int_{2 t-t^{\prime}}^{t}(t-s)^{\alpha-2}[X(s)-X(t)] d s .
\end{aligned}
$$

As $\alpha+h_{X}(t)>1$ we may choose an $\varepsilon>0$ small enough so that $\alpha+h_{X}(t)-\varepsilon>1$ and the first integral on the left hand side is finite. The second integral is of the order

$$
\begin{gathered}
\int_{2 t-t^{\prime}}^{t}(t-s)^{\alpha-2}[X(s)-X(t)] d s \\
=O\left(\int_{2 t-t^{\prime}}^{t}(t-s)^{\alpha+\left(h_{X}(t) \wedge 1\right)-2-\varepsilon} d s\right) \\
=O\left(\left|t^{\prime}-t\right|^{\alpha+\left(h_{X}(t) \wedge 1\right)-\varepsilon-1}\right) .
\end{gathered}
$$

For $k \geq 2$,

$$
\int_{0}^{2 t-t^{\prime}}(t-s)^{\alpha-k-1}[X(s)-X(t)] d s=d_{k}+\int_{t-\delta}^{2 t-t^{\prime}}(t-s)^{\alpha-1-k}[X(s)-X(t)] d s,
$$


where $d_{k}=\int_{0}^{t-\delta}(t-s)^{\alpha-1-k}[X(s)-X(t)] d s$. The second integral is of the order

$$
\begin{gathered}
\int_{t-\delta}^{2 t-t^{\prime}}(t-s)^{\alpha-1-k}[X(s)-X(t)] d s \\
=O\left(\int_{t-\delta}^{2 t-t^{\prime}}(t-s)^{\alpha+\left(h_{X}(t) \wedge 1\right)-1-k-\varepsilon} d s\right) \\
=O\left(\left|t^{\prime}-t\right|^{\alpha+\left(h_{X}(t) \wedge 1\right)-\varepsilon-k}\right) .
\end{gathered}
$$

The equations (3.21) and (3.27) yield

$$
J_{2}=C\left(t^{\prime}-t\right)+O\left(\left|t^{\prime}-t\right|^{\alpha+\left(h_{X}(t) \wedge 1\right)-\varepsilon}\right),
$$

where $C$ is a constant independent of $t^{\prime}$. Combining (3.8), (3.11), (3.14) and (3.28) we have

$$
Y\left(t^{\prime}\right)-Y(t)=P_{t}\left(t^{\prime}\right)+O\left(\left|t^{\prime}-t\right|^{\alpha+1}\right)+O\left(\left|t^{\prime}-t\right|^{\alpha+\left(h_{X}(t) \wedge 1\right)-\varepsilon}\right),
$$

where $P_{t}\left(t^{\prime}\right)$ is a local polynomial of $Y(t)$ and for any $\varepsilon>0$. Letting $\varepsilon \rightarrow 0$ we see that

$$
h_{Y}(t) \geq \min \left(\alpha+h_{X}(t), \alpha+1\right) .
$$

Theorem 1 Let $X(t)$ be the solution to the fractional integral equation (2.8) with $\beta_{n} \geq 1$ and let $L(t)$ a process with bounded sample paths. The singularity spectrum of $X(t)$ satisfies

$$
d_{X}(h)=d_{\mathcal{I}^{\beta_{n}-1} L}(h), \quad h \in\left[0,1+\beta_{n}-\beta_{n-1}\right) .
$$

Proof From our previous discussion on the solution of (2.8) we can say that, if $L(t)$ has bounded sample paths, then $X(t)$ will also have bounded sample paths. Clearly the local Hölder exponents of $\mathcal{I}^{\beta_{n}-1} L(t)$ and

$$
A_{n} X(t)+A_{n-1} \mathcal{I}^{\beta_{n}-\beta_{n-1}} X(t)+\ldots+A_{0} \mathcal{I}^{\beta_{n}-\beta_{0}} X(t)
$$

coincide by (2.8). As the local Hölder exponent of the sum of two functions is the infimum of the two, except perhaps when they are equal, in which case it may be larger, we may apply Lemma 1 to conclude that

$$
h_{X}(t)=h_{\mathcal{I}^{\beta_{n}-1} L}(t) \text {, whenever } h_{\mathcal{I}^{\beta_{n}-1} L}(t) \in\left[0,1+\beta_{n}-\beta_{n-1}\right) .
$$

If $h_{\mathcal{I}^{\beta_{n}-1} L}(t) \geq 1+\beta_{n}-\beta_{n-1}$, then $h_{X}(t) \geq 1+\beta_{n}-\beta_{n-1}$ and Theorem 1 follows.

Remark 1 A specific example of Theorem 1 is obtained when we let $L(t)$ be a Lévy motion. As the sample paths of all Lévy motions are right continuous with left limits, almost surely, they must be bounded on any finite interval $[0, T]$. When $\beta_{n}=1$ and $1 / \gamma<2-\beta_{n-1}$, where $\gamma$ is the upper index of the Lévy measure and $L(t)$ has no Brownian component, then Theorem 1 implies that the singularity spectrum of $X(t)$ is given by $(2.16)$.

When $\beta_{n}>1$ the right hand side of (2.8) is the fractional integral of Lévy motion for which we do not know the singularity spectrum. The following lemma provides an upper bound on the singularity spectrum. 
Lemma 2 Let $L(t)$ be a Lévy motion with upper index $\gamma \geq 1$ and no Brownian component. The singularity spectrum of the fractional integral of Lévy motion is bounded by

$$
d_{\mathcal{I}^{\beta} L}(h) \leq \gamma(h-\beta), \quad h<\beta+1 / \gamma .
$$

Proof The proof follows from Lemma 1 which gives $h_{\mathcal{I}^{\beta} L}(t) \geq \beta+h_{L}(t)$, the multifractal formalism (see Riedi [38], Section 7.1) and the form (2.16) of the singularity spectrum of Lévy motion.

\section{Semimartingale Representation}

An interesting problem in the development of a stochastic calculus for fractional differential equations is to determine when the solution has the semimartingale representation. Fractional Brownian motion, which is characterized by a spectral density of the form

$$
f(\lambda)=\frac{c}{|\lambda|^{2 \gamma}} \frac{\lambda^{2}}{1+\lambda^{2}}, c>0, \frac{1}{2}<\gamma<\frac{3}{2}, \lambda \in \mathbb{R}
$$

is known to be a semimartingale if and only if $\gamma=1$ (Lin [31]). Another dynamic model, fractional Riesz-Bessel motion, which is characterised by a spectral density of the form

$$
f(\lambda)=\frac{c}{|\lambda|^{2 \gamma}} \frac{1}{\left(1+\lambda^{2}\right)^{\alpha}} \frac{\lambda^{2}}{1+\lambda^{2}}, c>0, \frac{1}{2}<\gamma<\frac{3}{2}, \alpha>0, \lambda \in \mathbb{R}
$$

is known to be a semimartingale when $\alpha+\gamma \downarrow 3 / 2$, (Anh and Nguyen [4]). Using Lemma 1 , appropriate conditions on a fractional differential equation for its solution to be a semimartingale can be given.

Theorem 2 Let $X(t)$ be the solution to (2.3) with $L(t)$ being a Lévy motion whose Lévy measure has upper index $\gamma$ and without Brownian component. Then the following results hold:

(i) If $\beta_{n}<1$, then $X(t)$ is unbounded on any finite interval and thus not a semimartingale.

(ii) If $\beta_{n}>1$, then $X(t)$ is of null quadratic variation. Futhermore, if $\beta_{n}>2-1 / \gamma$, then $X(t)$ is of bounded 1-variation.

(iii) If $\beta_{n}=1$ and $\beta_{n-1}<1 / \gamma$, then $X(t)$ is a semimartingale.

Proof (i) By definition, $X(t)=\mathcal{D}^{1-\beta_{n}} Y(t)$, where $Y(t)$ is a process with a dense set of discontinuities, almost surely. It follows that $X(t)$ is unbounded on any finite interval and hence $X(t)$ is not a semimartingale.

(ii) For any sequence of partitions $\left\{\Delta_{n}\right\}$ of $[0, t]$ with $\left|\Delta_{n}\right| \rightarrow 0$ as $n \rightarrow \infty$, we have

$$
\begin{aligned}
E[X, X]_{t} & \leq \lim _{\left|\Delta_{n}\right| \rightarrow 0} \sum_{\left(t_{i+1}, t_{i}\right) \in \Delta_{n}} E\left(X\left(t_{i+1}\right)-X\left(t_{i}\right)\right)^{2} \\
& =\lim _{\left|\Delta_{n}\right| \rightarrow 0} \sum_{\left(t_{i+1}, t_{i}\right) \in \Delta_{n}} \int_{0}^{t_{i+1}}\left(G\left(t_{i+1}-s\right)-G\left(t_{i}-s\right)\right)^{2} d s \\
& \leq \lim _{\left|\Delta_{n}\right| \rightarrow 0} \sum_{\left(t_{i+1}, t_{i}\right) \in \Delta_{n}} \int_{-\infty}^{t_{i+1}}\left(G\left(t_{i+1}-s\right)-G\left(t_{i}-s\right)\right)^{2} d s .
\end{aligned}
$$


From Anh et al. [2] it is known that

$$
f(\omega)=\frac{1}{2 \pi}\left|\int_{0}^{\infty} e^{i \omega t} G(t) d t\right|^{2} \sim c|\omega|^{-2 \beta_{n}},
$$

as $\omega \rightarrow \infty$. It follows that for any $\varepsilon>0$,

$$
\begin{aligned}
E[X, X]_{t} & \leq \lim _{\left|\Delta_{n}\right| \rightarrow 0} C \sum_{\left(t_{i+1}, t_{i}\right) \in \Delta_{n}}\left|t_{i+1}-t_{i}\right|^{2 \beta_{n}-1-\varepsilon} \\
& \leq \lim _{\left|\Delta_{n}\right| \rightarrow 0} C\left|\Delta_{n}\right|^{2 \beta_{n}-2-\varepsilon} t \\
& =0 .
\end{aligned}
$$

A function will be of bounded 1-variation if and only if it is differentiable almost everywhere. Therefore, if the local Hölder exponent of $X(t)$ is greater than one almost everywhere, it will be of bounded 1-variation. From Lemma 1 the local Hölder exponent of $X(t)$ will be greater than one almost everywhere if $\beta_{n}>2-1 / \gamma$.

(iii) From (2.8) it is sufficient to show that

$$
B(t)=\frac{A_{n-1}}{A_{n}} \mathcal{I}^{1-\beta_{n-1}} X(t)+\ldots+\frac{A_{0}}{A_{n}} \mathcal{I}^{1-\beta_{0}} X(t)
$$

is of bounded 1-variation. Substituting $X(t)=-B(t)+L(t)$ into (4.8) and applying Lemma 1 , it is seen that this is equivalent to showing that $\mathcal{I}^{1-\beta_{n-1}} L(t)$ is of bounded 1 -variation. Using the same argument as in (ii) we conclude that $B(t)$ is of bounded 1-variation if $\beta_{n-1}<1 / \gamma$, and hence $X(t)$ is a semimartingale.

Remark 2 We can allow the Lévy motion in Theorem 2 to possess a Brownian component, in which case we set $\gamma=2$ regardless of the Lévy measure. Parts (ii) and (iii) of the theorem hold with the same proof if a Brownian component is included. For part (i) we note that $G(t) \sim C t^{\beta_{n}-1}$ as $t \rightarrow 0$ (see Anh et al. [2]). Applying Proposition 13 of Carmona et al. [13] shows that $X(t)$ will have no quadratic variation if $\beta_{n}<1$ and hence $X(t)$ is not a semimartingale for $\beta_{n}<1$.

Remark 3 As in the proof of Theorem 1, we do not make use of the assumption that $L(t)$ is a Lévy motion, only that it is a semimartingale with Hölder exponent $1 / \gamma$ almost everywhere. As all semimartingales have finite 2-variation, Lemma 4.3 of Dudley and Norvaiša [19] implies that semimartingales have a Hölder exponent greater than or equal to $1 / 2$ almost everywhere. Following the arguments of the proof of Theorem 2 (iii) we see that $X(t)$ will be a semimartingale if $\beta_{n}=1, \beta_{n-1}<1 / 2$ and $L(t)$ is a semimartingale.

\section{Prediction Formula}

Consider first the simple case of the fractional differential equation

$$
B \mathcal{D}^{1} X(t)+A \mathcal{D}^{\alpha} X(t)=\mathcal{D}^{1} L(t) .
$$

which was studied in Anh et al. [2]. It is assumed that we have observed the sample path of $X(t)$ from its initial condition $X(0)=0$ and so $\mathcal{F}_{t}=\sigma(X(s): 0 \leq s \leq t)$. To compute the conditional expectation $E\left(X(t+u) \mid \mathcal{F}_{t}\right)$ we can take advantage of the form of the solution as follows:

$$
E\left(X(t+u) \mid \mathcal{F}_{t}\right)=E\left(\frac{1}{B} L(t+u)-\frac{A}{B} \mathcal{I}^{1-\alpha} X(t+u) \mid \mathcal{F}_{t}\right)
$$




$$
\begin{gathered}
=E\left(\frac{1}{B}(L(t+u)-L(t))+X(t)-\frac{A}{B} \mathcal{I}^{1-\alpha} X(t+u)+\frac{A}{B} \mathcal{I}^{1-\alpha} X(t) \mid \mathcal{F}_{t}\right) \\
=X(t)+\frac{A}{B \Gamma(1-\alpha)} \int_{0}^{t}\left\{(t-s)^{-\alpha}-(t+u-s)^{-\alpha}\right\} X(s) d s \\
-\frac{A}{B \Gamma(1-\alpha)} \int_{t}^{t+u}(t+u-s)^{-\alpha} E\left(X(s) \mid \mathcal{F}_{t}\right) .
\end{gathered}
$$

Let $\phi(u ; t)=E\left(X(t+u) \mid \mathcal{F}_{t}\right)$ and

$$
\Phi(u ; t)=X(t)+\frac{A}{B \Gamma(1-\alpha)} \int_{0}^{t}\left\{(t-s)^{-\alpha}-(t+u-s)^{-\alpha}\right\} X(s) d s .
$$

Then

$$
\phi(u ; t)=\Phi(u ; t)-\frac{A}{B \Gamma(1-\alpha)} \int_{t}^{t+u}(t+u-s)^{-\alpha} \phi(s-t ; t) d s .
$$

A simple change of variable yields

$$
\phi(u ; t)=\Phi(u ; t)-\frac{A}{B} \mathcal{I}^{1-\alpha} \phi(u ; t) .
$$

Theorem 1.2 of Djrbashian [18] gives the solution to the above intregral equation as

$$
\phi(u ; t)=\Phi(u ; t)-\frac{A}{B} \int_{0}^{u}(u-v)^{-\alpha} E_{1-\alpha, 1-\alpha}\left(\frac{-A}{B}(u-v)^{1-\alpha}\right) \Phi(v ; t) d v .
$$

For the more general form of fractional differential equation (2.3) with $\beta_{n}=1$, the prediction formula is given by the solution to the integral equation

$$
\phi(u ; t)=\Phi(u ; t)-\frac{A_{n-1}}{A_{n}} \mathcal{I}^{1-\beta_{n-1}} \phi(u ; t)-\ldots-\frac{A_{0}}{A_{n}} \mathcal{I}^{1-\beta_{0}} \phi(u ; t),
$$

where

$$
\begin{aligned}
\Phi(u ; t)= & X(t)+\frac{A_{n-1}}{A_{n} \Gamma\left(1-\beta_{n-1}\right)} \int_{0}^{t}\left\{(t-s)^{-\beta_{n-1}}-(t+u-s)^{-\beta_{n-1}}\right\} X(s) d s+\ldots \\
& +\frac{A_{0}}{A_{n} \Gamma\left(1-\beta_{0}\right)} \int_{0}^{t}\left\{(t-s)^{-\beta_{0}}-(t+u-s)^{-\beta_{0}}\right\} X(s) d s,
\end{aligned}
$$

whose solution may be written as

$$
\phi(u ; t)=\Phi(u ; t)+\int_{0}^{u} G^{\prime}(u-v) \Phi(v ; t) d v,
$$

$G(t)$ being the Green function for the fractional differential equation

$$
\left(\mathcal{D}^{1}+\frac{A_{n-1}}{A_{n}} \mathcal{D}^{\beta_{n-1}}+\ldots+\frac{A_{0}}{A_{n}} \mathcal{D}^{\beta_{0}}\right) X(t)=\delta(t) .
$$




\section{Numerical Approximation of Sample Paths}

This section will provide a numerical scheme to approximate the sample paths of equations of the form (2.3). We first show that their Green function is completely monotonic on $[0, \infty)$ if $\beta_{n}=1$. Bernstein's theorem then implies that this Green function can be written in the form

$$
G(t)=\int_{0}^{\infty} e^{-\lambda t} \mu(d \lambda),
$$

where $\mu(d \lambda)$ is a finite Borel measure on $[0, \infty)$ (Feller [22]). In view of (2.5) and (6.1), the solution of $(2.3)$ with $\beta_{n}=1$ is then given by

$$
X(t)=\int_{0}^{t} \int_{0}^{\infty} e^{-\lambda(t-s)} \mu(d \lambda) d L(s) .
$$

It should be noted that $\int_{0}^{t} e^{-\lambda(t-s)} d L(s)$ is the solution of the Orstein-Uhlenbeck-type equation

$$
d Y(\lambda, t)=-\lambda Y(\lambda, t) d t+d L(t), Y(\lambda, 0)=0 .
$$

We will follow Carmona et al. [13] and Camona and Coutin [12] which give a fast and efficient algorithm for simulating Gaussian LRD processes based on the representation

$$
X(t)=\int_{0}^{t} \int_{0}^{\infty} e^{-\lambda(t-s)} \mu(d \lambda) d B(s),
$$

where $\mu(d \lambda)$ is a Borel measure satisfying

$$
\int_{0}^{\infty} \frac{1}{1+\lambda^{1 / 2}} \mu(d \lambda)<\infty
$$

and $B(t)$ is a Brownian motion. They prove mean-square and almost-sure convergence in this case. An example of a process with representation (6.4) is a type II fractional Brownian motion. When $(6.1)$ is square integrable on $[0, \infty)$ the process $(6.4)$ is not stationary, but does converge in mean square to a stationary Gaussian process. Furthermore, (6.4) can be made stationary by letting the initial condition in (6.3) be a Gaussian random function on $[0, \infty)$ with mean zero and covariance function

$$
E\left[Y(\lambda, 0) Y\left(\lambda^{\prime}, 0\right)\right]=\frac{\sigma^{2}}{\lambda+\lambda^{\prime}} .
$$

We will show that the solution to a certain class of FDEs has the representation (6.2). In this case we extend the almost sure convergence result to include Lévy motion as a driving term.

\subsection{Approximation algorithm}

The following approximation scheme is adapted from Carmona et al. [13]. Define a compact set $K \subset[0, \infty)$ by $K=\left[r^{-m}, r^{n}\right]$ with $m, n$ being positive integers and $r>1$. Denote the geometric partition of $K$ by $\pi=\left\{A_{i}\right\}$ with $A_{i}=\left[r^{i}, r^{i+1}\right), i=$ $-m, \ldots, n-1$. Consider the following approximation of $X(t)$ :

$$
X_{\pi}(t)=\sum_{\pi} \mu\left\{\left[r^{i}, r^{i+1}\right)\right\} Y\left(r^{i}, t\right),
$$


where $Y\left(r^{i}, t\right)$ is the solution to the Ornstein-Uhlenbeck-type equation

$$
d Y(\lambda, t)=-\lambda Y(\lambda, t) d t+d L(t)
$$

subject to the initial condition $Y(\lambda, 0)=0$. Finally, the Ornstein-Uhlenbeck-type process is approximated by

$$
Y_{\Delta}(t)=L(t)
$$

for $0<t \leq \Delta$ and

$$
Y_{\Delta}(t)=e^{-\lambda(t-(n-1) \Delta)} Y_{\Delta}((n-1) \Delta)+L(t)-L((n-1) \Delta),
$$

for $(n-1) \Delta<t \leq n \Delta$. The approximation of $X(t)$ is then

$$
X_{\pi, \Delta}(t)=\sum_{\pi} Y_{\Delta}\left(r^{i}, t\right) \mu\left\{\left[r^{i}, r^{i+1}\right)\right\}
$$

\subsection{Representation as sums of OU-type processes}

For the approximation (6.11) to be useful in simulating sample paths of FDEs, we need to determine if the Green function has the representation (6.1). While it is clear that this cannot be the case for $\beta_{n}>1$ as $G(0)=0$, the Green function will have the representation (6.1) if $\beta_{n}=1$. For this we need part (iii) of Theorem 5.10 from Inoue [26] (see also its Theorems 1.1 and 1.2).

Theorem 3 Let $R(t)$ be the covariance function of a stationary process satisfying

$$
R(t)=\int_{0}^{\infty} e^{-\lambda|t|} \sigma(d \lambda)
$$

where $\sigma(d \lambda)$ is a finite Borel measure on $(0, \infty)$. Then

$$
\frac{1}{R(0)} \int_{0}^{\infty} e^{i \zeta t} R(t) d t=\left(-i \zeta-i \zeta \int_{0}^{\infty} e^{i \zeta t} \gamma(t) d t\right)^{-1}
$$

where

$$
\gamma(t)=1_{(0, \infty)}(t) \int_{0}^{\infty} e^{-\lambda t} \rho(d \lambda)
$$

and $\rho(d \lambda)$ is a Borel measure satisfying

$$
\int_{0}^{\infty} \frac{1}{1+\lambda} \rho(d \lambda)<0, \quad \int_{0}^{\infty} \frac{1}{\lambda} \rho(d \lambda)=\infty
$$

Theorem 4 The Green function of a fractional differential equation is completely monotonic on $[0, \infty)$ if $\beta_{n}=1$.

Proof With $\beta_{n}=1$, the Green function of (2.3) has the Laplace transform

$$
\begin{aligned}
g(p) & =\frac{1}{\left(A_{n} p+\ldots+A_{1} p^{\beta_{1}}+A_{0} p^{\beta_{0}}\right)} \\
& =\frac{1}{A_{n}}\left(p+p \sum_{i=0}^{n-1} \frac{A_{i}}{A_{n}} p^{\beta_{i}-1}\right)^{-1}
\end{aligned}
$$


(Podlubny [37], p. 158). Let

$$
\rho(d \lambda)=\sum_{i=0}^{n-1} \frac{A_{i} \sin \left(\pi \beta_{i}\right)}{A_{n} \pi} \lambda^{\beta_{i}-1} d \lambda .
$$

Clearly, (6.18) satisfies (6.15) if $\beta_{0}>0$ and has Laplace transform

$$
\gamma(t)=\sum_{i=0}^{n-1} \frac{A_{i} t^{-\beta_{i}}}{A_{n} \Gamma\left(1-\beta_{i}\right)} .
$$

Hence letting $\zeta=i p$ and $R(0)=A_{n}^{-1}$ in (6.13) we see that $g(p)$ is the Laplace transform of a function which is completely monotonic on $[0, \infty)$ for $\beta_{0}>0$. For $\beta_{0}=0$, the same approach is followed with the exception of using Theorem 8.5 of Okabe [34] instead of Theorem 5.10 of Inoue [26]. This completes the proof.

It is not sufficient to know that we can write the Green function in the form (6.1). We need to know the measure $\mu(d \lambda)$. For this purpose, we rely on the following Theorem 1.3-5 of Djrbashian [18] for the two-parameter Mittag-Leffler function, which can be defined by the series expansion

$$
E_{\alpha, \beta}(x)=\sum_{k=0}^{\infty} \frac{z^{k}}{\Gamma(\alpha k+\beta)}, z \in \mathbb{C}, \alpha>0, \beta>0 .
$$

It is noted that

$$
\begin{gathered}
E_{1,1}(z)=e^{z}, E_{2,1}(z)=\cosh \sqrt{z}, E_{2,2}(z)=(\sinh \sqrt{z}) / \sqrt{z} \\
E_{1,2(z)}=\left(e^{z}-1\right) / z, E_{1,3}(z)=\left(e^{z}-1-z\right) / z^{2}
\end{gathered}
$$

and

$$
E_{1 / 2,1}(z)=e^{z^{2}} \operatorname{erf}(-z), \operatorname{erf}(z)=\frac{2}{\sqrt{\pi}} \int_{z}^{\infty} e^{-t^{2}} d t .
$$

Theorem 5 If $\rho<1$ and $\mu \in(0,1+\rho)$, then the following formula is true:

$$
E_{\rho, \mu}\left(-x^{\rho}\right) x^{\mu-1}=\frac{1}{\pi} \int_{0}^{\infty} \frac{\sin (\pi(\mu-\rho))+\tau^{\rho} \sin (\pi \mu)}{1+2 \cos (\pi \rho) \tau^{\rho}+\tau^{2 \rho}} \tau^{\rho-\mu} e^{-x \tau} d \tau, \quad x \in(0, \infty) .
$$

We now consider some examples of fractional differential equations whose Green function has the representation (6.1).

Example 1 The Green function of the two-term fractional differential equation

$$
B \mathcal{D}^{\beta} X(t)+A \mathcal{D}^{\alpha} X(t)=\delta(t)
$$

is

$$
G(t)=\frac{1}{B} t^{\beta-1} E_{\beta-\alpha, \beta}\left(-\frac{A}{B} t^{\beta-\alpha}\right) \mathbf{1}_{(0, \infty)}(t)
$$

(see Anh et al. [2]). If $\beta \leq 1$, then simple algebra yields

$$
G(t)=\frac{1}{\pi} \int_{0}^{\infty} \frac{A \lambda^{\alpha} \sin (\alpha \pi)+B \lambda^{\beta} \sin (\beta \pi)}{B^{2} \lambda^{2 \beta}+A^{2} \lambda^{2 \alpha}+2 A B \cos (\pi(\beta-\alpha)) \lambda^{\beta+\alpha}} e^{-t \lambda} d \lambda .
$$


This example includes a number of important cases. If $\alpha=0$ then we have the fractional relaxation equation (Podlubny [37]). When $\alpha>0$ the resulting process is long-range dependent and will also possess second-order intermittency if $\beta<1$. The case of $\beta=1$ and $\alpha<1 / \gamma$ results in a semimartingale.

Example 2 Following the description in Okabe [35], we consider a sphere of mass $m$ and radius $r$ moving in a fluid with viscosity $\eta$ and density $\rho$. Denoting the velocity of the sphere by $X(t)$, the random force by $W(t)$ and the drag force by $F(t)$, Newton's second law reads

$$
m \frac{d X(t)}{d t}=-F(t)+W(t) .
$$

Solving a linearised Navier-Stokes equation subject to incompressibility and sticky boundary conditions we have

$$
F(t)=6 \pi r \eta X(t)+\frac{2}{3} \pi r^{3} \rho \frac{d X(t)}{d t}+6 \pi r^{2} \sqrt{\frac{\rho \eta}{\pi}} \int_{-\infty}^{t}(t-s)^{-1 / 2} \frac{d X(s)}{d s} d s .
$$

Newton's equation then becomes the Stokes-Boussinesq-Langevin equation

$$
m^{*} \frac{d X(t)}{d t}=-6 \pi \rho \eta X(t)-6 \pi r^{2} \sqrt{\frac{\rho \eta}{\pi}} \int_{-\infty}^{t}(t-s)^{-1 / 2} \frac{d X(s)}{d s}+W(t),
$$

where $m^{*}=m+\frac{2}{3} \pi r^{3} \rho$ is the effective mass of the sphere. If $W(t)$ is white noise, that is if $W(t)=\frac{d B(t)}{d t}$ (understood in the random distribution sense), where $B(t)$ is Brownian motion, then the solution to the Stokes-Boussinesq-Langevin equation has the moving average representation

$$
X(t)=\int_{-\infty}^{t} G(t-u) d B(u),
$$

where the kernel $G(t)$ has the representation

$$
G(t)=\frac{\alpha}{\pi} \frac{C_{B}}{m^{*}} \int_{0}^{\infty} e^{-t \lambda} \frac{\sqrt{\lambda}}{\left(\beta_{B}-\lambda\right)^{2}+C_{B}^{2} \lambda} d \lambda, \quad t>0
$$

with

$$
C_{B}=\frac{6 \pi r^{2} \sqrt{\rho \eta}}{m^{*}}, \beta_{B}=\frac{6 \pi r \sqrt{r \eta}}{m^{*}} .
$$

\subsection{Almost sure convergence}

We first prove the rate of convergence for the approximation to an Ornstein-Uhlenbecktype process.

Lemma 3 Let $q>1$ and let $p$ be such that $q^{-1}+p^{-1}>1$. Assume $L(t)$ is of bounded $p$-variation, the difference between the Ornstein-Uhlenbeck-type process and its approximation defined by $(6.9-6.10)$ is uniformly bounded on $[0, T]$ by

$$
\|L(T)\|_{(p)}\left(C_{1} \lambda \Delta+C_{2}(\lambda \Delta)^{1-1 / q}\right),
$$

almost surely, for some finite positive constants $C_{1}, C_{2}$. 
Proof Let $t=n \Delta$ then we may write (6.10) as the Moore-Pollard-Stieltjes (MPS) integral

$$
Y_{\Delta}(n \Delta)=\int_{0}^{n \Delta}\left(\sum_{m=1}^{n} e^{-\lambda \Delta(n-m)} I\{s \in((m-1) \Delta, m \Delta]\}\right) d L(s)
$$

(see Dudley and Norvaiša [19] for details). The difference between $Y_{\Delta}(n \Delta)$ and $Y(n \Delta)$ is the MPS integral $\int_{0}^{n \Delta} f_{\Delta}(s) d L(s)$, where

$$
f_{\Delta}(s)=\sum_{m=1}^{n} e^{-\lambda \Delta(n-m)} I\{s \in((m-1) \Delta, m \Delta]\}-e^{\lambda(n \Delta-s)} .
$$

By elementary arguments the $q$-variation, $q>1$, of $f_{\Delta}(s)$ is

$$
\begin{aligned}
v_{q}\left(f_{\Delta}\right) & =2 \sum_{m=1}^{n} e^{-q \lambda \Delta m}\left(1-e^{-\lambda \Delta}\right)^{q} \\
& \leq 2(\Delta \lambda)^{q} \sum_{m=1}^{n} e^{-q \lambda \Delta m} \\
& \leq 2(\lambda \Delta)^{q} \frac{1}{1-e^{-q \lambda \Delta}}
\end{aligned}
$$

It follows that

$$
\|f\|_{[q]} \leq 2^{1 / q} \lambda \Delta\left(\frac{1}{1-e^{-q \lambda \Delta}}\right)^{1 / q}+1-e^{-\lambda \Delta} \leq C\left((\lambda \Delta)^{1-1 / q}+\lambda \Delta\right)
$$

for some finite constant $C$. Applying the Love-Young inequality to the difference of $Y(n \Delta)$ and $Y_{\Delta}(n \Delta)$ we get

$$
\left|Y_{\Delta}(n \Delta)-Y(n \Delta)\right| \leq C\left((\lambda \Delta)^{1-1 / q}+\lambda \Delta\right)\|L(n \Delta)\|_{(p)},
$$

for some finite constant $C$. When $(n+1) \Delta \geq t>n \Delta$ then from (6.10),

$$
\left|Y_{\Delta}(t)-Y(t)\right| \leq\left|Y(n \Delta)-Y_{\Delta}(n \Delta)\right|+\left|\int_{n \Delta}^{t}\left(e^{-\lambda(t-s)}-1\right) d L(s)\right| .
$$

Applying the (6.32) and the Love-Young inequality to (6.33) we get the difference $\left|Y(t)-Y_{\Delta}(t)\right|$ is bounded by

$$
C\left((\lambda \Delta)^{1-1 / q}+\lambda \Delta\right)\|L(n \Delta)\|_{(p)}+\lambda \Delta\|L(t)-L(n \Delta)\|_{(p)} .
$$

Clearly this is bounded by (6.25) and proof is completed.

Theorem 6 Suppose that $K_{N}$ is a sequence of compact sets growing to $(0, \infty), r_{N} \rightarrow$ 1 and $\Delta_{N} \rightarrow 0$ such that

$$
\Delta_{N}^{1-1 / q} \int_{K_{N}} \lambda \mu(d \lambda) \rightarrow 0, \int_{K_{N}^{C}} \mu(d \lambda) \rightarrow 0
$$


as $N \rightarrow \infty$. Then, almost surely,

$$
\sup _{t \leq T}\left|X(t)-X_{\pi, \Delta}(t)\right| \rightarrow 0
$$

Proof We first consider the error in approximating $X(t)$ by $X_{K_{N}}(t)$ $=\int_{K_{N}} Y(\lambda, t) \mu(d \lambda)$ :

$$
\begin{aligned}
\left|X(t)-X_{K_{N}}(t)\right| & =\left|\int_{K^{C}} \int_{0}^{t} e^{-\lambda(t-s)} d L(s) \mu(d \lambda)\right| \\
& \leq 2 C_{1, p}\|L(t)\|_{(p)} \int_{K_{N}^{C}} \mu(d \lambda),
\end{aligned}
$$

where the inequality is derived using the Love-Young inequality. We now consider the error in approximating $X_{K}(t)$ by $X_{\pi}(t)$ :

$$
\begin{aligned}
\left|X_{K_{N}}(t)-X_{\pi}(t)\right| & =\left|\int_{K} Y(\lambda, t) \mu(d \lambda)-\sum_{\pi} \mu\left\{\left[r_{N}^{i}, r_{N}^{i+1}\right)\right\} Y\left(r_{N}^{i}, t\right)\right| \\
& \leq \sum_{\pi} \sup _{\lambda \in A_{i}}\left|Y\left(r_{N}^{i}, t\right)-Y(\lambda, t)\right| \mu\left\{\left[r_{N}^{i}, r_{N}^{i+1}\right)\right\}
\end{aligned}
$$

For any $\lambda_{2}>\lambda_{1}$,

$$
\begin{aligned}
\left|Y\left(\lambda_{1}, t\right)-Y\left(\lambda_{2}, t\right)\right| & =\left|\int_{0}^{t}\left(e^{-\lambda_{1}(t-s)}-e^{-\lambda_{2}(t-s)}\right) d L(s)\right| \\
& =\left|\int_{0}^{t} e^{-\lambda_{1}(t-s)}\left(1-e^{-\left(\lambda_{2}-\lambda_{1}\right)(t-s)}\right) d L(s)\right| \\
& \leq 2 C_{1, p} e^{-\lambda_{1} t}\left(1-e^{-\left(\lambda_{2}-\lambda_{1}\right) t}\right)\|L(t)\|_{(p)},
\end{aligned}
$$

where (6.43) is an application of the Love-Young inequality. Now,

$$
\left|X_{K_{N}}(t)-X_{\pi}(t)\right| \leq 2 C_{1, p}\|L(t)\|_{(p)} \sum_{\pi} e^{-r_{N}^{i} t}\left(1-e^{-r_{N}^{i}\left(r_{N}-1\right) t}\right) \mu\left\{\left[r_{N}^{i}, r_{N}^{i+1}\right)\right\} .
$$

Using the inequality $\left|1-e^{-r_{N}^{i}\left(r_{N}-1\right) t}\right| \leq r_{N}^{i}\left(r_{N}-1\right) t,(6.44)$ becomes

$$
\begin{aligned}
\left|X_{K_{N}}(t)-X_{\pi}(t)\right| \leq & \leq 2 C_{1, p} \\
& \|L(t)\|_{(p)}\left(r_{N}-1\right) \sum_{\pi} r_{N}^{i} t e^{-r_{N}^{i} t} \mu\left\{\left[r^{i}, r^{i+1}\right)\right\} \\
& \leq 2 C_{1, p}\|L(t)\|_{(p)}\left(r_{N}-1\right) \int_{K_{N}} \mu(d \lambda) .
\end{aligned}
$$

Finally, we need the error in approximating $X_{\pi}(t)$ by $X_{\pi, \Delta}(t)$ :

$$
\left|X_{\pi}(t)-X_{\pi, \Delta}(t)\right| \leq \sum_{\pi} \mu\left\{\left[r_{N}^{i}, r_{N}^{i+1}\right)\right\}\left|Y_{\Delta}\left(r_{N}^{i}, t\right)-Y\left(r_{N}^{i}, t\right)\right| .
$$


Applying Lemma 3 yields

$$
\begin{gathered}
\left|X_{\pi}(t)-X_{\pi, \Delta}(t)\right| \leq C\|L(t)\|_{(p)} \sum_{\pi} \mu\left\{\left[r_{N}^{i}, r_{N}^{i+1}\right)\right\}\left(\left(r_{N}^{i} \Delta\right)^{1-1 / q}+r_{N}^{i} \Delta\right)(6.4 \\
\leq C\|L(t)\|_{(p)}\left(\int_{K_{N}} \lambda \mu(d \lambda)\right) \Delta_{N}^{1-1 / q}
\end{gathered}
$$

for some finite constant $C$. Using the triangle inequality we may combine equations (6.38), (6.46) and (6.49) to conclude that the almost sure convergence holds if the conditions of the theorem are satistfied. This completes the proof.

Remark 4 In Example 1, if $\beta_{n}<1$, then $\int_{K_{N}^{C}} \mu(d \lambda) \nrightarrow 0$ and so a.s. convergence does not hold. However, mean-square convergence still holds by the results of Carmona et al. [13].

\subsection{Simulations}

Sample paths of the fractional differential equation

$$
\mathcal{D} X(t)+\mathcal{D}^{\beta_{0}} X(t)=\mathcal{D} L(t)
$$

were generated in MATLAB using the algorithm described in Subsection 6.1. The simulations were carried out for three different types of Lévy motion: $\alpha$-stable, inverse Gaussian and normal inverse Gaussian.

A common way to define an $\alpha$-stable random variable is by its characteristic function:

$$
\psi(z)=\left\{\begin{array}{cc}
\exp \left\{-\sigma^{\alpha}|z|^{\alpha}(1-i \beta \operatorname{sign}(z) \tan (\alpha \pi / 2))+i \mu z\right\}, & \alpha \in(0,1) \cup(1,2], \\
\exp \{-\sigma|z|+i \mu z\}, & \alpha=1,
\end{array}\right.
$$

where $\alpha$ is the index of stability, $\sigma$ is the scale parameter, $\beta$ is the skewness parameter and $\mu$ is the shift parameter. A way to simulate random variables from the symmetric stable distribution with unit scale parameter is as follows (see Janiki and Weron [29]):

(a) Generate $V$ from a uniform distribution on $[-\pi / 2, \pi / 2]$ and $W$ from an exponential distribution with mean 1.

(b) Compute

$$
X=\frac{\sin (\alpha V)}{\cos ^{1 / \alpha}(V)}\left(\frac{\cos (V-\alpha V)}{W}\right)^{\frac{1-\alpha}{\alpha}} .
$$

Sample paths of the corresponding Lévy motion can then be generated by

$$
L(n h)=\sum_{i=1}^{n} h^{1 / \alpha} X_{i}
$$

where $h$ is the time step.

The density function of an inverse Gaussian random variable $I G(\delta, \gamma)$ is of the form $g(u)=(\gamma / \delta)^{-1 / 2}\left[2 K_{-1 / 2}(\delta \gamma)\right]^{-1} u^{-3 / 2} \exp \left\{-\frac{1}{2}\left(\frac{\delta^{2}}{u}+\gamma^{2} u\right)\right\} \mathbf{1}_{(0, \infty)}(u), \delta>0, \gamma \geq 0$, 
where

$$
K_{\lambda}(z)=\frac{1}{2} \int_{0}^{\infty} s^{\lambda-1} \exp \left\{-\frac{1}{2}\left(s+\frac{1}{s}\right) z\right\} d s, \quad z \geq 0
$$

is the modified Bessel function of the third kind with index $\lambda$. Random variables from the inverse Gaussian distribution can be generated by using the method described in Seshadri [41]:

(a) Generate $Y$ from a $\chi_{1}^{2}$ distribution.

(b) Set $m_{1}=\gamma / \delta, m_{2}=\delta^{2}$ and

$$
X_{1}=m_{1}+\frac{m_{2}^{2} Y}{2 m_{2}}-\frac{m_{1}}{2 m_{2}} \sqrt{4 m_{1} m_{2} Y+m_{1}^{2} Y^{2}}
$$

(c) Generate $U$ from a uniform distribution on $[0,1]$. If $U \leq \frac{m_{1}}{m_{1}+X}$ set $X=X_{1}$, otherwise set $X=\frac{m_{1}^{2}}{X_{1}}$.

Sample paths of the corresponding Lévy motion can then be generated by

$$
L(n h)=\sum_{i=1}^{n} X_{i}\left(\delta h, \gamma h^{2}\right),
$$

where the $X_{i}(\delta, \gamma)$ are $I G(\delta, \gamma)$ distributed.

The density function of the normal inverse Gaussian random variable $N I G(\alpha, \beta, \delta, \mu)$ is given by

$$
g(u)=\frac{\alpha}{\pi} \exp \left\{\delta \sqrt{\alpha^{2}-\beta^{2}}+\beta(u-\mu)\right\} \frac{K_{1}(\delta \alpha g(u-\mu))}{g(u-\mu)}, \quad u \in \mathbb{R},
$$

where $\delta>0,0 \leq|\beta| \leq \alpha, \mu \in \mathbb{R}, g(x)=\sqrt{\delta^{2}+x^{2}}$. The distribution is symmetric around $\mu$ provided $\beta=0$. The parameters of the distribution centered around zero have the following meaning: $\alpha$ is the steepness parameter, $\beta$ the asymmetry parameter and $\delta$ the scale parameter. The normal distribution $N(\mu, \beta)$ is the limiting case with $\beta=0, \alpha \rightarrow \infty$ and $\delta / \alpha=b$, and the Cauchy distribution is the limiting case of $N I G(\alpha, 0,0,1)$ with $\alpha \rightarrow 0$. Random variables from the normal inverse Gaussian distribution can be generated by simulating an $I G(\delta, \alpha)$ random variable $\sigma^{2}$, a standard normal random variable $\varepsilon$ and compute $X=\sigma \varepsilon$. The random variable $X$ will be distributed as $N I G(\alpha, 0, \delta, 0)$ and the Lévy motion can by constructed by

$$
L(n h)=\sum_{i=1}^{n} X_{i}\left(\alpha h^{2}, \delta h\right),
$$

where the $X_{i}(\alpha, \delta)$ are $N I G(\alpha, 0, \delta, 0)$ distributed.

The three cases of (i) $\alpha$-stable with $\alpha=1.9$ and scale parameter equal to one, (ii) inverse Gaussian with $\delta=1, \gamma=1$ and (iii) normal inverse Gaussian with $\alpha=1$, $\beta=0, \delta=1, \mu=0$ were considered. In each case, the order of fractional derivative $\beta_{0}$ was set to 0.33 . The parameters for the algorithm were set to $n=m=24, \Delta=0.01$ and $r=1.1$. The results are presented in Figures 1-3 for the three cases respectively. 


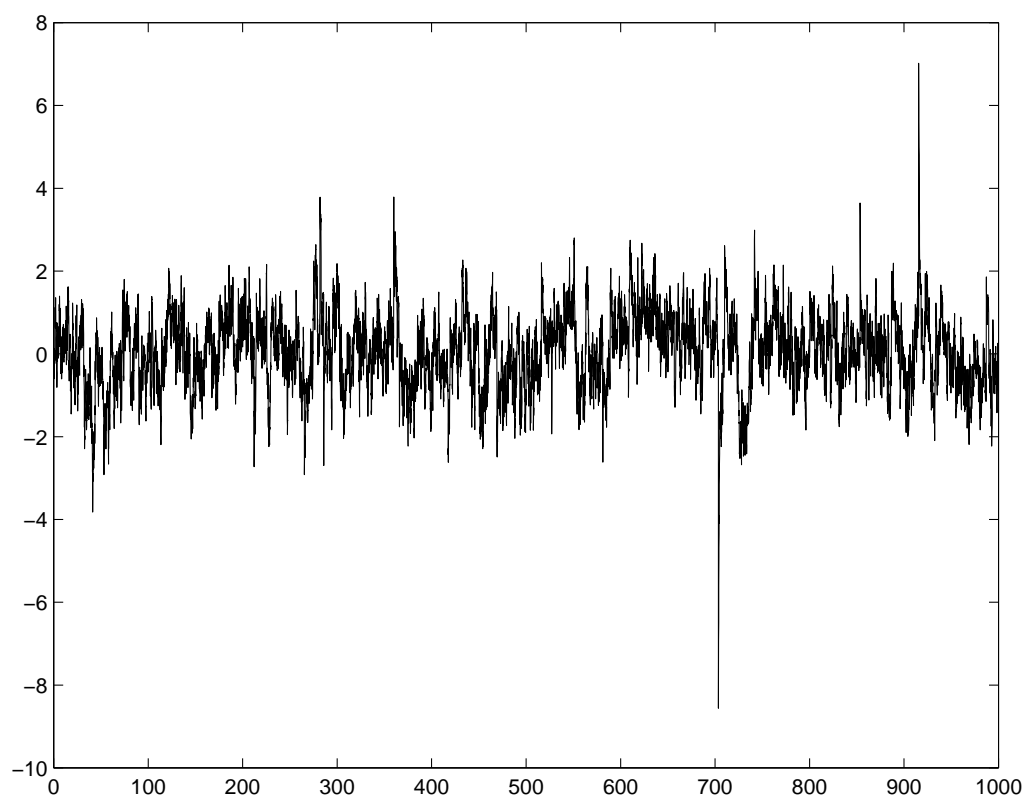

Figure 1: A sample path of the two-term fractional differential equation driven by the generalized derivative of an $\alpha$-stable process

\section{References}

[1] Anh, V.V., Angulo, J.M. and Ruiz-Medina, M.D., Possible long-range dependence in fractional random fields, J. of Stat. Plan. and Infer. 80:1/2 (1999), 95-110.

[2] Anh, V.V., Heyde, C.C., and Leonenko, N.N., Dynamic models of long-memory processes driven by Lévy noise with applications to finance and macroeconomics, J. Appl. Prob. 39:4 (2002), 730-747.

[3] Anh, V.V. and Leonenko, N.N., Scaling laws for fractional diffusion-wave equation with singular initial data, Stats. and Prob. Letters 48 (2000), 239-252.

[4] Anh, V.V. and Nguyen, C.N., Semimartingale representation of fractional Riesz-Bessel motion, Finance and Stoch. 5 (2001), 83-101.

[5] Baillie, R.T., Long memory processes and fractional integration in econometrics, J. of Econometrics 73 (1996), 5-59.

[6] Barndorff-Nielson, O., Superpositions of ornstein-uhlenbeck type processes. Research Report No. 2, MaPhySto, Aarhus Univ., 1999.

[7] Barndorff-Nielsen, O.E., Processes of normal inverse Gaussian type, Finance and Stoch. 2 (1998), 41-68.

[8] Barndorff-Nielsen, O.E. and Shephard, N., Aggregation and model construction for volatility models, Working Paper Series No. 10, Center for Analytical Finance, Univ. of Aarhus, 1998.

[9] Barndorff-Nielsen, O.E. and Shephard, N. Shephard, Incorporation of a leverage effect in a stochastic volatility model, Research Report No. 18, MaPhySto, Univ. of Aarhus, 1998.

[10] Bibby, M. and Sørensen, M., A hyperbolic diffusion model for stock prices, Finance and Stoch. 1 (1997), 24-41. 


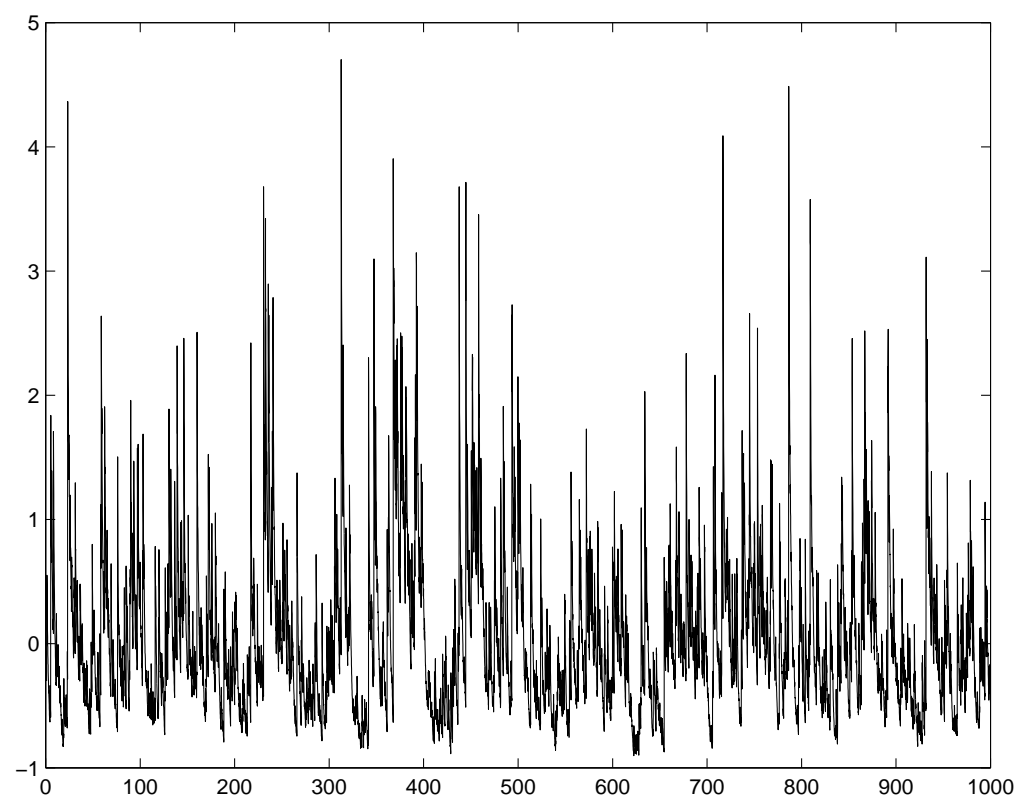

Figure 2: A sample path of the two-term fractional differential equation driven by the generalized derivative of an inverse Gaussian Lévy process

[11] Bretagnolle, J., $p$-variation de functions aléatoires, 2eme partie: processes à accroissements indépendants. In Séminaire de Probabilités IV, volume $\mathbf{2 5 8}$ of Lecture Notes in Mathematics, Springer, Berlin 1972.

[12] Carmona, P. and Coutin, L., Fractional Brownian motion and the Markov property. Electronic Commun. in Prob. 3 (1998), 97-107.

[13] Carmona, P., Coutin, L., and Montsenty, G., Applications of a representation of long memory Gaussian processes, 1998. Preprint.

[14] Chambers, M.J., The estimation of continuous parameter long-memory time series models, Econometric Theory 12 (1996), 374-390.

[15] Comte, F. and Renault, E., Long memory continuous-time models, J. Econometrics $\mathbf{7 3}$ (1996), 101-149.

[16] Comte, F. and Renault, E., Long memory in continuous-time stochastic volatility models, Math. Finance 8 (1998), 291-323.

[17] Ding, Z. and Granger, C.W.J., Modelling volatility persistence of speculative returns: a new approach, J. of Econometrics 73 (1996), 185-215.

[18] Djrbashian, M.M., Harmonic Analysis and Boundary Value Problems in Complex Domain, Birkhäuser Verlag, Basel 1993.

[19] Dudley, R.M. and Norvaiša, R., An Introduction To p-Variation and Young Integrals, Lecture Notes, MaPhySto 1998.

[20] Eberlein, E. and Keller, U., Hyperbolic distributions in finance. Bernoulli 1:3 (1995), 281-299.

[21] Eberlein, E. and Raible, S., Term structure models driven by general Lévy processes. Math. Finance 9 (1999), 31-53. 


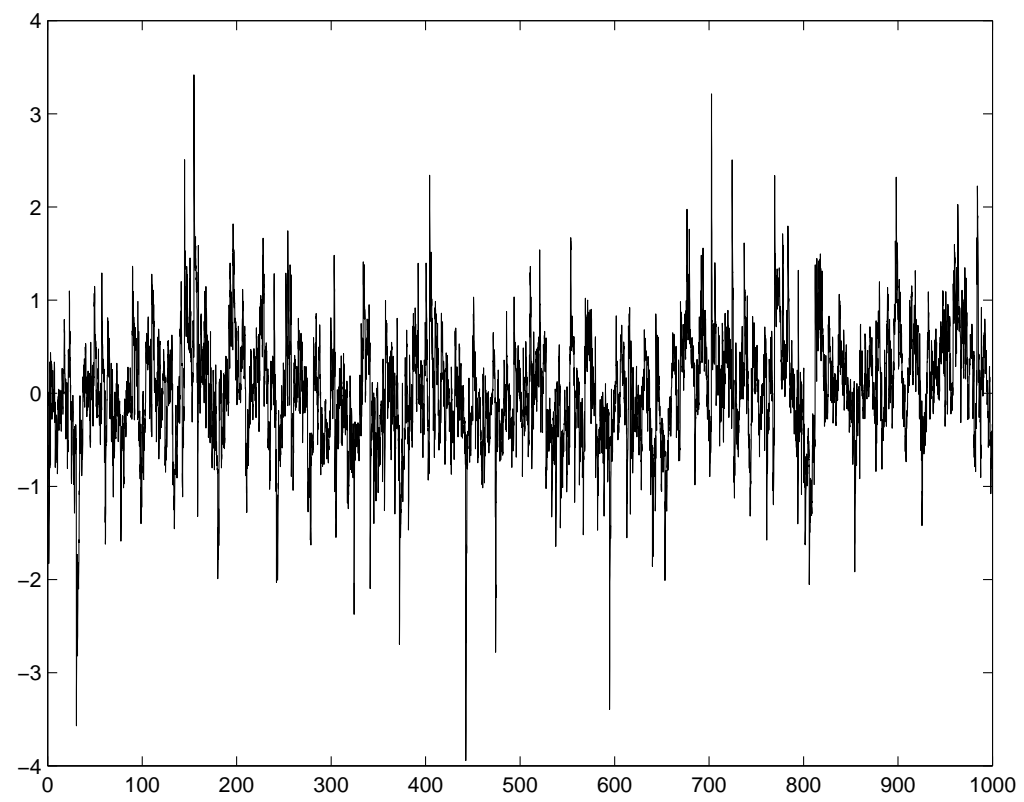

Figure 3: A sample path of the two-term fractional differential equation driven by the generalized derivative of aa normal inverse Gaussian Lévy process

[22] Feller, W., An Introduction to Probability Theory and its Applications II, Wiley, New York 1971.

[23] Gay, R. and Heyde, C.C., On a class of random field models which allows long range dependence. Biometrika 77 (1990), 401-403.

[24] Granger, C.W.J. and Ding, Z., Varieties of long-memory models, J. of Econometrics 73 (1996), 61-72.

[25] Iglói, E. and Terdik, G., Long-range dependence through gamma-mixed OrnsteinUhlenbeck process, Elect. J. of Prob. 4 (1999), 1-33.

[26] Inoue, A., On the equations of stationary processes with divergent diffusion coefficients, J. Fac. Sci. Univ. Tokyo, Sect. IA 40 (1993), 307-336.

[27] Jaffard, S., Multifractal formalism for functions, part 1: results valid for all functions, SIAM J. Math. Anal. 28 (1997), 944-970.

[28] Jaffard, S., The multifractal nature of Lévy proesses, Probability Theory and Related Fields (114) (1999), 207-227.

[29] Janiki, A. and Weron, A., Simulation and Chaotic Behaviour of Alpha Stable Stochastic Processes, Marcel Dekker 1994.

[30] Kress, R., Linear Integral Equations 82 of Applied Mathematical Sciences, Springer-Verlag, New York 1989.

[31] Lin, S.J., Stochastic analysis of fractional Brownian motions. Stoch. and Stoch. Rep. $\mathbf{5 5}$ (1995), 121-140.

[32] Mandelbrot, B.B., Intermittent turbulence in self-similar cascades: divergence of high moments and dimension of the carrier, J. Fluid Mech. 62 (1974), 331-358.

[33] Mueller, C., The heat equation with Lévy noise. Stoch. Proc. and their Appl. 74 (1998), $67-82$. 
[34] Okabe, Y., On KMO-Langevin equations for stationary Gaussian processes with Tpositivity, J. Fac. Sci. Univ. Tokyo 33 (1986), 1-56.

[35] Okabe, Y., Langevin equation and fluctuation-dissipation theorem, In: Stochastic Processes and their Applications in Mathematics and Physics (edited by S. Albeverio, P. Blanchard, and L. Streit), Kluwer Academic Publishers 1990.

[36] Oppenheim, G. and Viano, M.-C., Obtaining long-memory by aggregating random coefficients of discrete and continuous time simple short memory processes, Pub IRMA 49:(V) (1999), 1-16.

[37] Podlubny, I., Fractional Differential Equations, Academic Press, San Diego 1999.

[38] Riedi, R.H., Multifractal processes, Technical Report TR99-06, ECE Dept., Rice University 1999.

[39] Rydberg, T.H., Generalized hyperbolic diffusion process with applications in finance, Math. Finance 9:2 (1999), 183-201.

[40] Samko, S.G., Kilbas, A.A., and Marichev, O.I., Fractional Integrals and Derivatives, Gordon and Breach Science Publishers 1993.

[41] Seshadri, V., The Inverse Gaussian Distribution: A Case Study in Exponential Families, Oxford University Press 1993.

[42] Viano, M.C., Deniau, C., and Oppenheim, G., Continuous-time fractional ARMA processes, Stats. and Prob. Letters 21 (1994), 323-336.

[43] Young, L.C., An inequality of the Hölder type, connected with Stiltjes integration, Acta Mathematica 67 (1936), 251-282. 


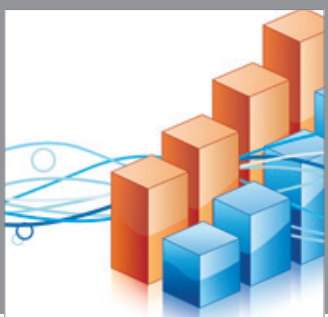

Advances in

Operations Research

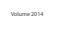

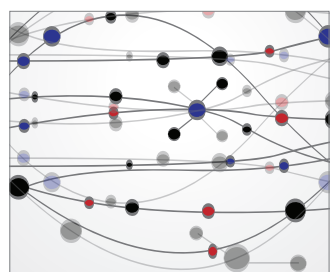

\section{The Scientific} World Journal
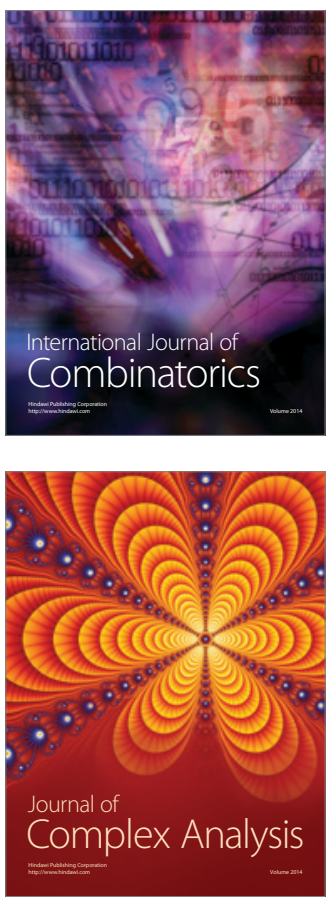

International Journal of

Mathematics and

Mathematical

Sciences
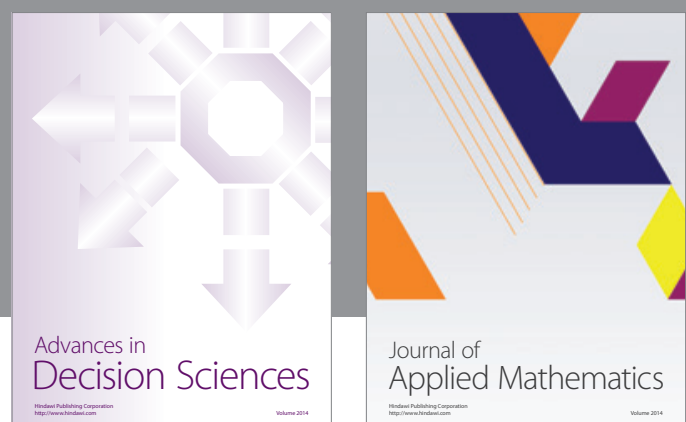

Journal of

Applied Mathematics
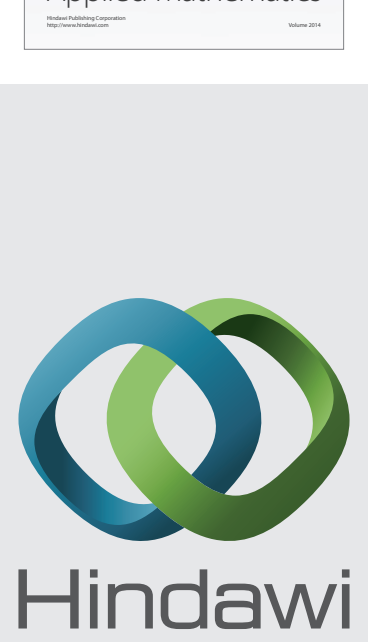

Submit your manuscripts at http://www.hindawi.com
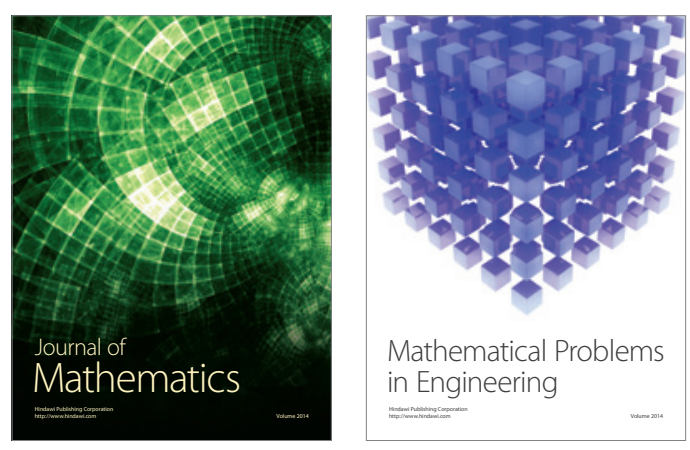

Mathematical Problems in Engineering
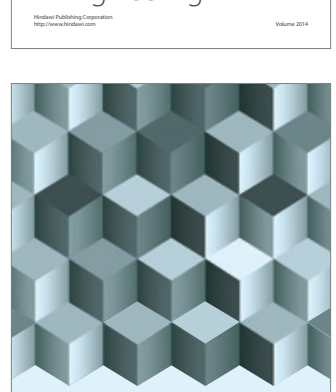

Journal of

Function Spaces
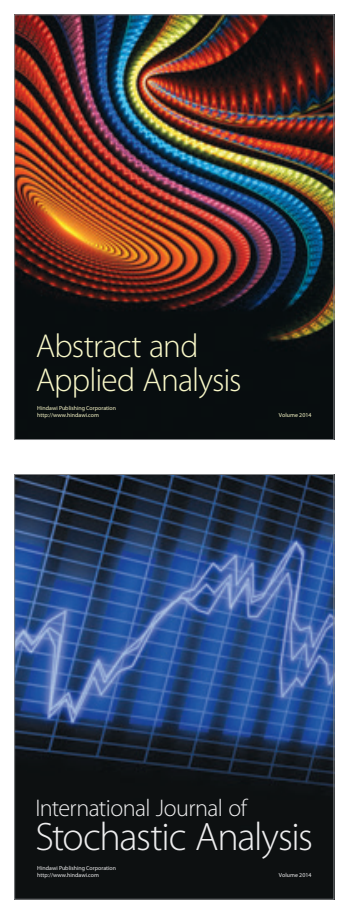

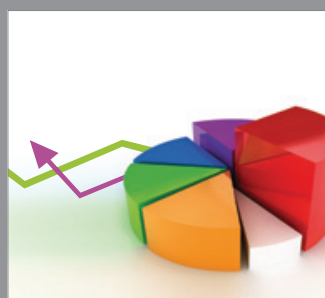

ournal of

Probability and Statistics

Promensencen
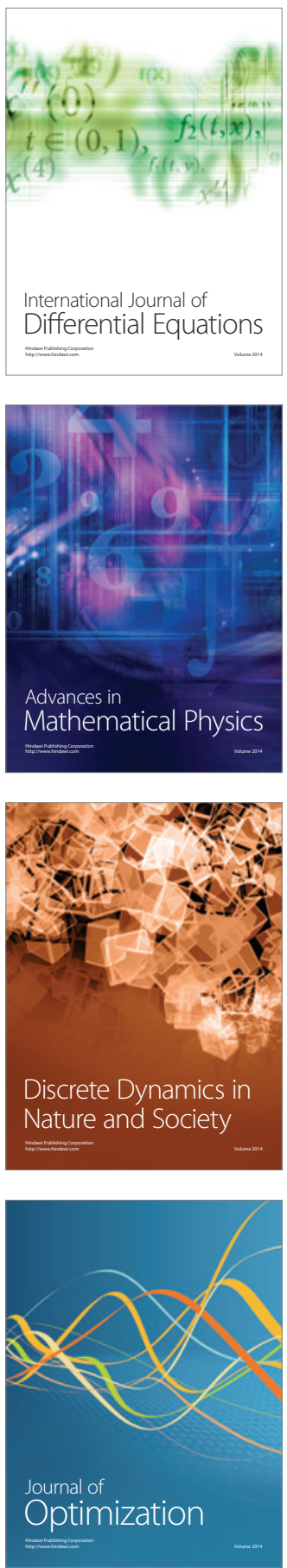